\title{
Capacitance Sensor System for Oligonucleotide Hybridization Detection
}

\author{
Jang Zern Tsai*, Ching Jung Chen, Wen Yih Chen ${ }^{1}$, \\ Jen Tsai Liu ${ }^{1}$ and Yue Ming Hsin \\ Department of Electrical Engineering, National Central University, \\ 300 Jhongda Rd., Jhongli, Taoyuan County 32001, Taiwan \\ 'Department of Chemical Engineering and Material Engineering, National Central University, \\ 300 Jhongda Rd., Jhongli, Taoyuan County 32001, Taiwan
}

(Received January 5, 2010; accepted May 28, 2010)

Key words: capacitance, detection, interdigitated chip, oligonucleotide, biosensor

In this paper, we describe an electronic method of oligonucleotide detection implemented in silicon chips. The detection system includes an interdigitated chip and capacitance detection circuit. Interdigitated microelectrodes were microfabricated with a width of $10 \mu \mathrm{m}$, a gap of $3 \mu \mathrm{m}$, and a total length of 3,320 $\mu \mathrm{m}$. Oligonucleotide detection was tested on an electrode surface precoated with a functional monolayer by a self-assembly technique. In this study, oligonucleotide detection used the fundamental hybridization of the complementary strand. To enhance the capacitance change in oligonucleotide detection, we used the sandwich conjugation method with the $\mathrm{Au}$ nanoparticles cross-linked at the end of a single DNA strand. The detection system was designed to automatically measure the individual capacitances of oligonucleotides detected from the array chip. The capacitance of a biomolecule was measured using comb-shaped electrodes and, due to DNA hybridization, the capacitance increased by $20.31 \%$. In this work, we successfully developed a multipoint capacitance sensor that offers the potential to detect different sequences in the future.

\section{Introduction}

Many types of biosensor are under development for the detection of various biological molecules. The detection of oligonucleotides has revolutionized biological science, and the DNA hybridization detection format is gaining favor. In this approach, a single DNA strand binds to a complementary stranded DNA, and hybridization occurs. The translation of the hybridization formation into a measurable signal can be performed using an electrochemical detector, ${ }^{(1,2)}$ a frequency response system, ${ }^{(3)}$ or an optical method. ${ }^{(4)}$ Recently, the general clinical approach for oligonucleotide diagnosis assay

${ }^{*}$ Corresponding author: e-mail: jztsai@ee.ncu.edu.tw 
has been based on fluorescence detection. ${ }^{(5)}$ However, optical instruments are more expensive and complex to implement, and the optical detection method usually uses fluorescence labeling, which is quenched, depending on the chemical excitation lifetime. Thus, the development of a microelectronic system, which has fast response, high sensitivity, and high selectivity for oligonucleotide detection, is one of the best strategies.

Nucleic acid hybridization is an important oligonucleotide technology, and nucleic acid with a solid conducting surface interaction has been extensively investigated. Electrochemical impedance spectroscopy (EIS) is the main tool for investigating processes that occur at a conducting solid/electrolyte interface. ${ }^{(1,2)}$ Electrochemical techniques use many different types of electrode and geometry for impedimetric measurement, especially interdigitated electrodes. These have two significant advantages: (1) a high interelectrode capacitance allows the measurement of dielectric properties and (2) the electric fields of the cell geometry allow micromolecular detection. $^{(6)}$

In this study, we describe a capacitance sensor for the detection of nucleic acid DNA hybridization on silicon chips. Because the target oligonucleotide is complementary to the capture and probe strands at the end of Au nanoparticles, it specifically recognizes contiguous elements and enhances the electric capacity of oligonucleotides attached to $\mathrm{Au}$ nanoparticles. In principle, we have evaluated the electrical properties of DNAmodified nanoparticles, provided that a sufficient number of particles can attach to the chip surface and give a measurable electrical signal. The interdigitated electrodes were fabricated by standard photolithography. The chip has an inert substrate coated on two comb electrodes. The inert substrate can be chosen by different methods, according to a set of molecules to be detected. When we apply an excitation sinusoidal signal to the interdigitated electrodes, the capacitance change of the sensor depends on the difference in dielectric permittivity between the comb electrodes.

Thus, detecting DNA hybridization by this method can be achieved by measuring the capacitance changes using the interdigitated comb-shaped electrodes and the capacitance detection system.

\section{Research Methodology}

\subsection{Preparation of interdigitated chip}

The sensing chip contains an array of interdigitated metallic electrode pairs with an interelectrode distance of $3 \mu \mathrm{m}$. It was fabricated by photolithography on a p-type silicon wafer with a (100) orientation and a $120 \mathrm{~nm}$ layer of silicon oxide deposited by low-pressure chemical vapor deposition (LPCVD). The array chip was built with the following three metallic layers: the first layer (metal 1) is the row-line connection, the second layer (metal 2) is the column-line connection, and the third layer (metal 3) is the top layer of the interdigitated electrodes. A BCB dielectric layer inserted between metal 1 and metal 2 reduces the parasitic capacitance that can affect its sensitivity to DNA capacitance. Figure 1 shows a cross section of the chip. All fabrication processes followed the photolithographic technologies, including photoresist spinning, UV 


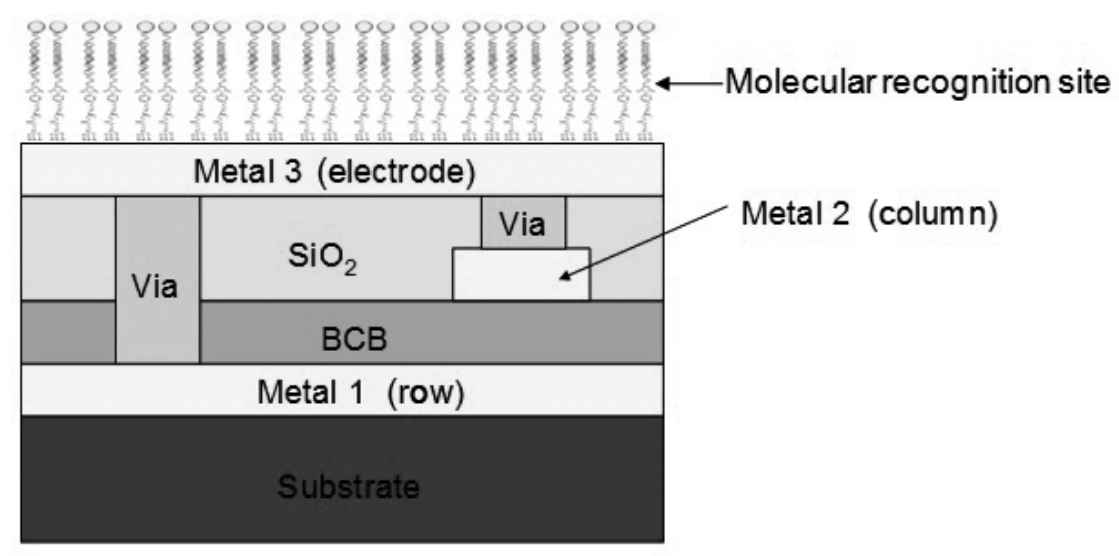

Fig. 1. Cross-sectional diagram of the interdigitated sensing chip.

light exposure, and pattern development; $\mathrm{Cr} / \mathrm{Au}$ was then deposited by E-gun thermal evaporation and the materials were etched with a high-density plasma etcher.

\subsection{Surface modification}

A self-assembled monolayer ( $\mathrm{SAM}$ ) was formed in the $\mathrm{SiO}_{2}$ region of the chip surface. The monolayer immobilizaction process followed the method described previously. ${ }^{(7)}$ Briefly, the chip was immersed in a trimethoxysilylpropyldiethylenetria mine (DETA) acidic methanol solution $\left(0.15 \mathrm{M}\right.$ acetic acid containing $3.7 \% \mathrm{H}_{2} \mathrm{O}$ and $2.3 \%$ DETA). When the chip surface was coated by DETA, the DETA was provided the amino groups to be attached with succinimidyl 4-[malemidophenyl] butyrate (SMPB). Then, the DETA-modified chip was immersed in a $1 \mathrm{mM}$ SMPB solution (ethanol: $\mathrm{DMSO}=80: 20$ ) for $2 \mathrm{~h}$ at room temperature. After $2 \mathrm{~h}$, the chip was immersed in $1 \mu \mathrm{M}$ capture DNA, overnight at room temperature. The SMPB has an amine group; therefore, it can be attached to the 5 ' end of thiolated capture DNA. ${ }^{(8,9)}$

The DNA hybridization processes in this investigation were of the sandwich type. Deoxyribonucleic acid (DNA) samples were purchased from MDBio (Taiwan). Their base sequences were as follows: capture DNA: 5'-TAACAATAATCCTTTTTTTTTTSH-3'; target DNA: 5'-GGATTATTGTTAAATATTGATAAGGAT-3'; probe DNA: 5' -SH-TTTTTTTTTTATCCTTATCAATATT-3'. When the capture DNA hybridizes with the target DNA, the probe DNA hybridizes with the target strand and gold nanoparticles are attached to the probe sequences to increase their capacitance sensitivity ${ }^{(10,11)}$ (see Fig. 2).

The DNA immobilization was characterized by contact angle and atomic force microscopy (AFM) observations. The contact angle is an invaluable parameter for understanding material surface properties, and AFM provides a true three-dimensional surface profile and does not require any special treatment that would irreversibly change or damage the sample. 


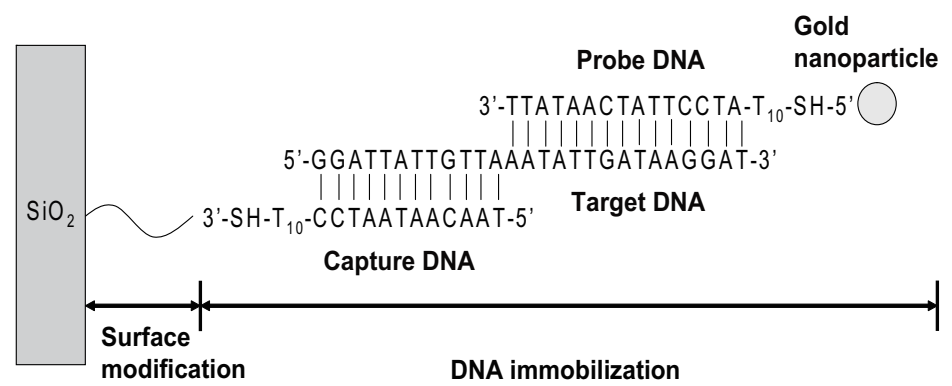

Fig. 2. Biomolecular structure of hybridized DNA on the silicon chip.

\subsection{Detection system fabrication}

To measure the DNA, we designed a simple circuit to record small capacitance signals for oligonucleotide detection. This system used a multiplexer to select the appropriate detection unit of the interdigitated chip. The excitation sinusoidal signal was applied through an audio transformer and generated a full voltage. If a voltage $\left(V_{\text {pulse }}\right)$ was applied at the interdigitated electrode, the oligonucleotide capacitance $\left(C_{\text {sensor }}\right)$ was determined using a low-pass filter from an operational amplifier. With the oligonucleotide attached to the chip, the capacitance of the output signal would change $\left(C_{\text {sensor }}\right)$, indicating that hybridization took place. In this circuit, the feedback capacitance stores a charge transported by a current pulse; the output voltage amplitude is proportional to the charge. To easily discriminate the varying capacitances, we used an RMS-to-DC circuit to convert the analog signal into a DC voltage and restricted the dc output from 0 to $5 \mathrm{~V}$ with a level shift circuit. Finally, a signal converter IC (ADC080X) and an 8,051 microprocessor assisted in signal detection for real-time monitoring (Fig. 3).

\section{Results and Discussion}

\subsection{Physical properties of the chip}

Each interdigitated electrode had a total length of 3,320 $\mu \mathrm{m}$ (Au patterning), and the finger gaps were optimized at $3 \mu \mathrm{m}$. An array sensor with 441 comb-shaped electrodes was designed and tested. The capacitance of interdigitated electrode pairs was determined with an HP4284 LCR meter at about $101 \mathrm{fF}$ at $1 \mathrm{kHz} .^{(12)}$ In the array chip, the comb-shaped electrodes are in parallel and connected to nine interdigitated electrode pairs as a sensing unit to enhance the capacitive value for easy oligonucleotide detection. The whole array consists of $7 \times 7$ units and can be used to detect the capacitance changes of DNA hybridization and nonhybridization. Figure 4 shows a top-view photograph of an array with the comb-shaped electrodes; the inset graph shows an array sensor with 49 sensing units bonded on the PCB board. 


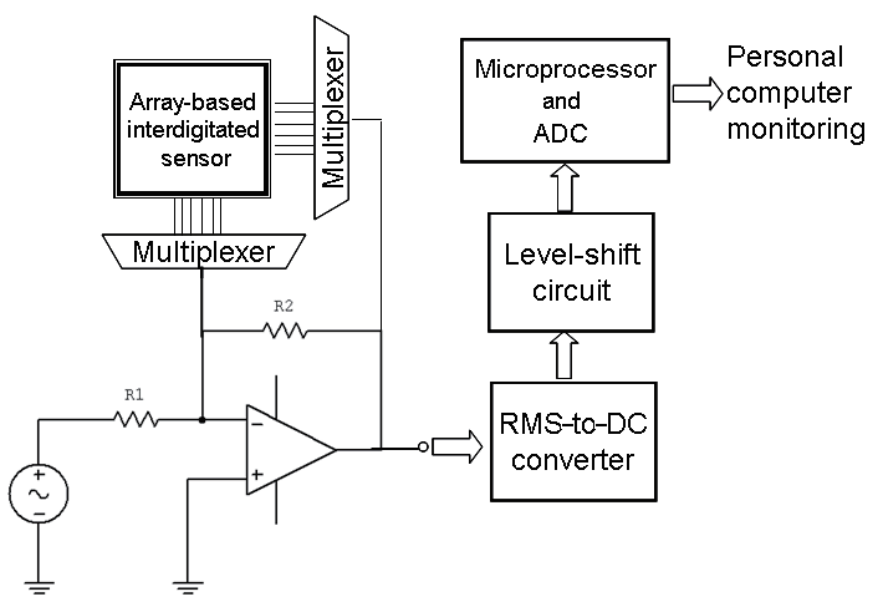

Fig. 3. Block diagram of the capacitance detection system.

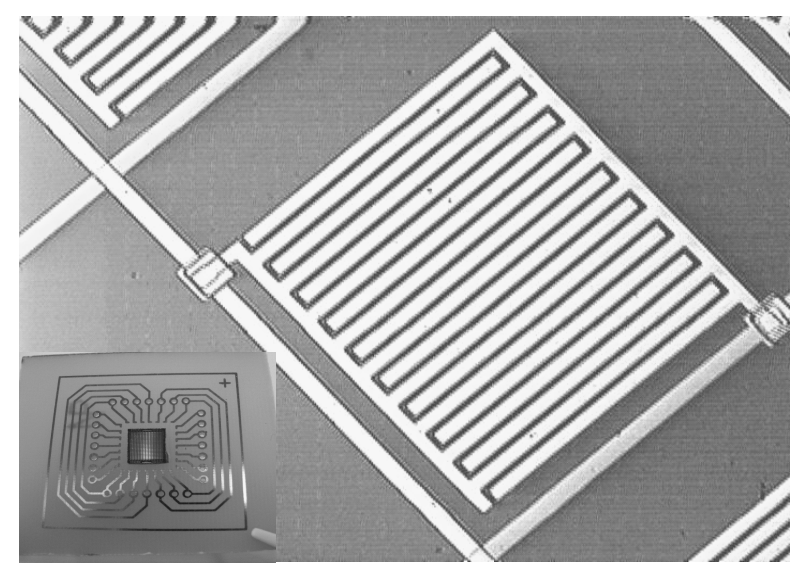

Fig. 4. Interdigitated sensing unit of the array chip. The inset shows a sensor array bonded on a PCB board.

\subsection{Surface modification for oligonucleotide detecting}

From the surface functionalization, after the final process, the chip has a silicon oxide surface that covalently bonds with oligonucletides. In the preliminary identification, to understand the surface characterization of the functionalization monolayer, we used the contact angle to verify the difference in the modification layer on the $\mathrm{SiO}_{2}$ surface. The initial contact angle of bare $\mathrm{SiO}_{2}$ was about $6.18^{\circ}$ because of the $\mathrm{OH}$ group on the chip surface. After forming the DETA monolayer, the contact angle changed to 
$43.47^{\circ}$, which was attributed to the DETA layer of the amine group, which was more hydrophobic than the bare $\mathrm{SiO}_{2}$ surface. Finally, the contact angle increased to 54.29 owing to the $N$-hydroxysuccinimide reactive groups of the SMPB layer, which became more hydrophobic than a DETA layer. The measurement result is close to the theoretical value and, after modification, the functionalization monolayer matched the design. The DNA hybridization processes in this investigation are designed as a sandwich type; the 5 ' end of the thiolated capture DNA was attached to the SMPB layer. The results are shown in Fig. 5; the direct relationship between DNA hybridization and nonhybridization with the deposition of gold nanoparticles was clearly established by AFM. A visual analysis showed the DNA hybridization and nonhybridization with the Au nanoparticles attached. The results show that the Au nanoparticle coverages of DNA hybridization and nonhybridization were about $68 \pm 10 \%$ (Fig. 5(a)) and $12 \pm 7 \%$ (Fig. 5(b)), respectively. This indicated the different morphologies on the chip surface and that DNA hybridization successfully covered the chip surface. By this method, the Au nanoparticles provide different electric conductivity properties for capacitance changes of oligonucletide detection.

(a)

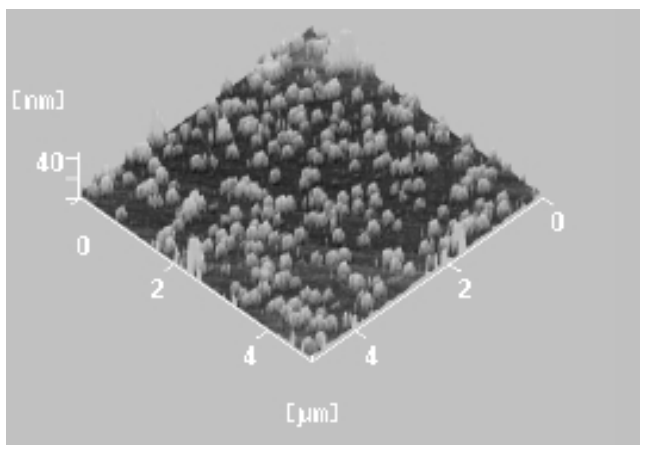

(b)

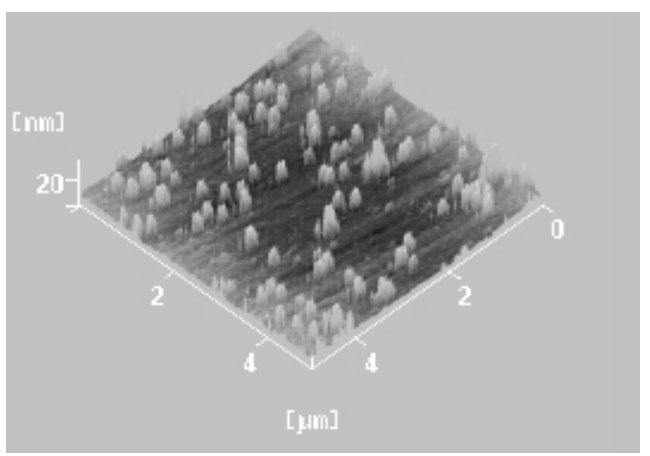

Fig. 5. AFM images of the interelectrode area: (a) hybridization surface and (b) nonhybridization surface. 
(a)
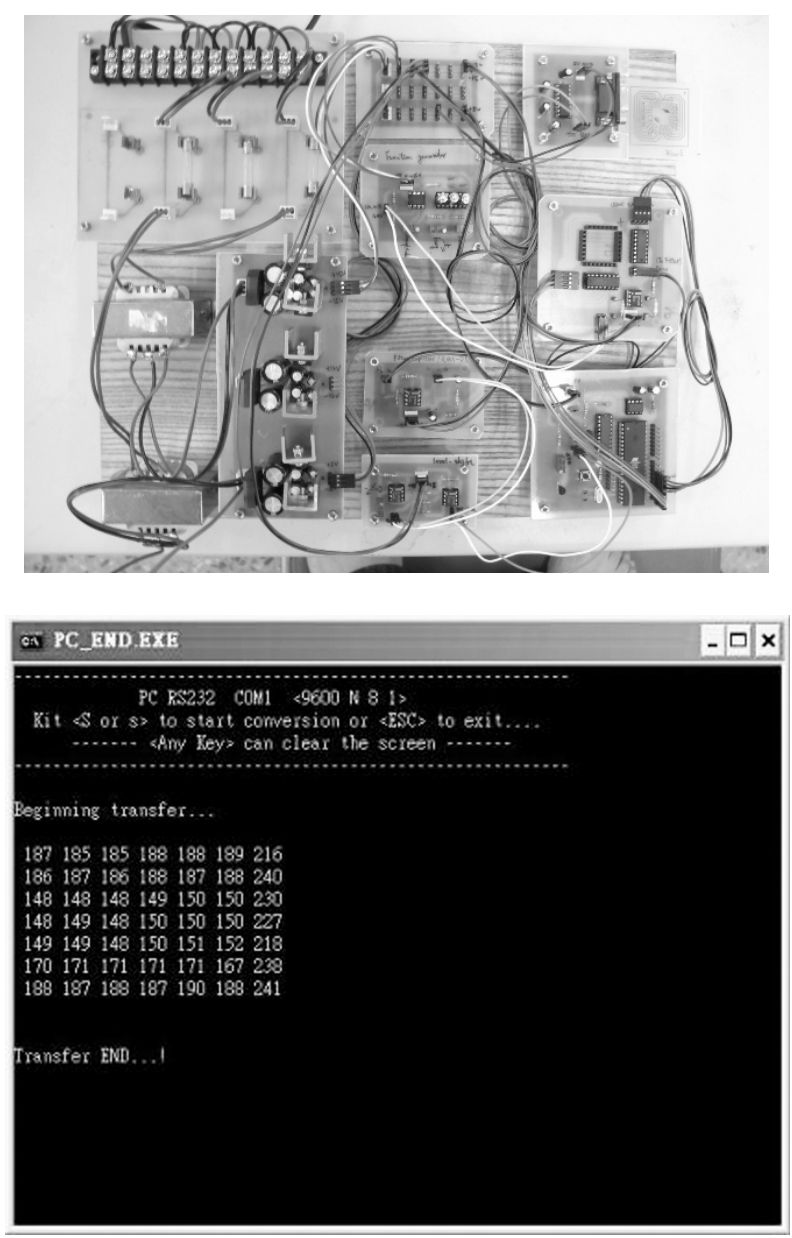

Fig. 6. Capacitance measurement using the detection system: (a) hardware of the measurement system and (b) capacitance signal displayed on the monitor.

\subsection{Automatic capacitance detection system}

To measure the capacitance change from DNA hybridization, an automatic detection system was fabricated with a dedicated circuit. This detection system includes a power supply, a sinusoidal generator circuit, a capacitance readout circuit, and a multiplexer controller. Figure 6(a) shows a photograph of the capacitance detection system with a microprocessor IC and a peripheral circuit. The capacitance values were recorded using the PC and automatically monitored using the 8,051 microprocessor with a $\mathrm{C}^{++}$language program, as shown in Fig. 6(b). The detector simplifies the display of the capacitance response of DNA hybridization dielectric characteristics. Figure 7 shows the signal used 


\section{Hybridization}

(a)

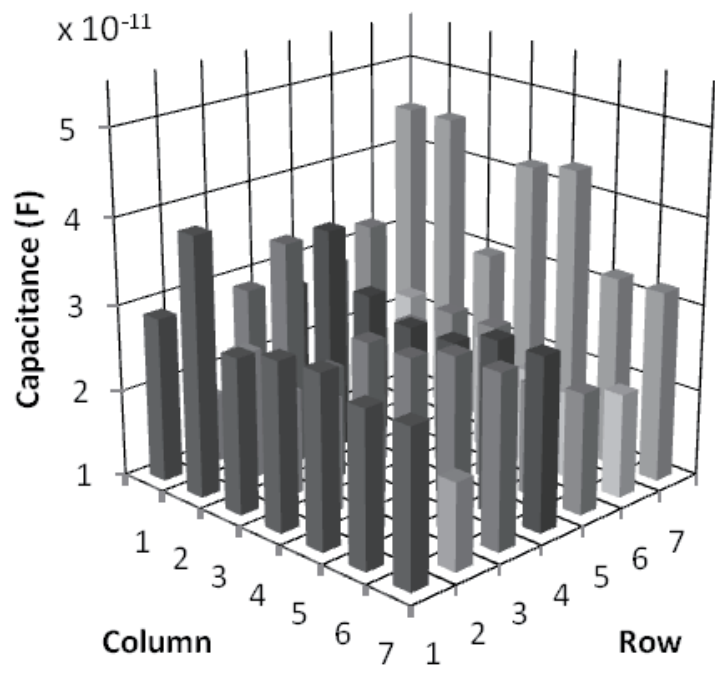

\section{Nonhybridization}

(b)

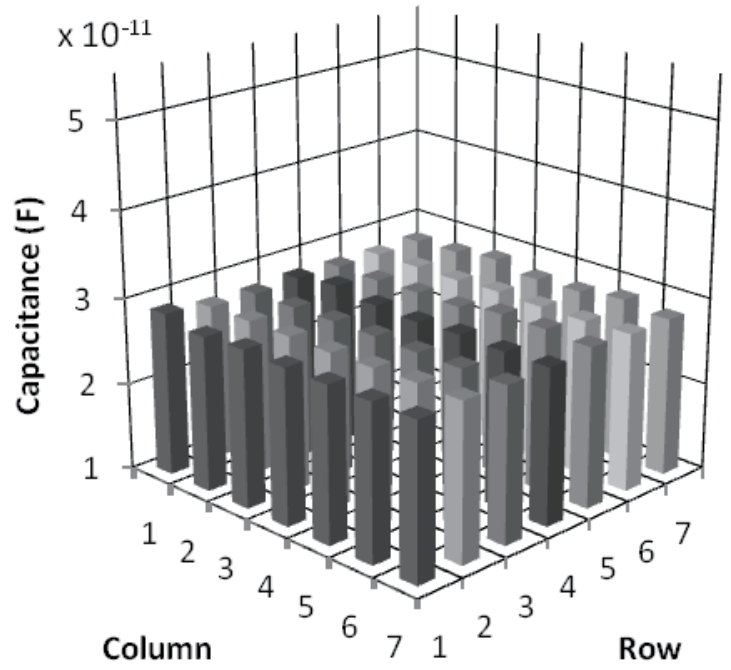

Fig. 7. Measured capacitances of the interdigitated electrode array for DNA (a) hybridization and (b) nonhybridization monitoring. 
by this detection system that monitors DNA hybridization and nonhybridization with different interdigitated electrode units in the array chip. The monitor of the detection result included the 49 data of an array chip for each test. The experimental data greater than threefold calculations showed that the capacitance values increased owing to the DNA hybridization process, as shown in Fig. 7(a). The values are smaller than those in Fig. 7(b), which shows the capacitance values of DNA nonhybridization, namely, the average capacitance was about $24.89 \mathrm{pF}$ and its standard deviation was about \pm 2.83 , and the DNA hybridization average capacitance was about $31.25 \mathrm{pF}$ and its standard deviation was about \pm 5.4 . The results proved highly reproducible through a series of detections. Until we obtained the statistics result of the capacitance detection value, the hybridization and nonhybridization threshold values remained $30.1 \mathrm{pF}$. Hybridization increases the capacitance by about $20.31 \%$.

\section{Conclusions}

We developed a novel detection system with an interdigitated array sensor for DNA hybridization detection. The experimental results show that the capacitance change at the interdigitated electrode chip can increase by as much as $20.31 \%$ when hybridization occurs. Our capacitance detection technique presented in this work enables very well controlled oligonucleotide hybridization detection and shows a high-speed response detection time. The utilization of our user friendly interface designed system can provide a convenient method for oligonucleotide hybridization detection. Moreover, the capacitance array chip investigated in this study is suitable for a possible integration with a microfabricated system, and it can be implemented in standard CMOS processes with signal processing circuits to create a Si-based biosensor chip in the future.

\section{References}

1. M. Yi, K. H. Jeong and L. P. Lee: Biosens. Bioelectron. 20 (2005) 1320.

2. W. Cai, J. R. Peck, D. W. Van der Weide and R. J. Hamers: Biosens. Bioelectron. 19 (2004) 1013.

3. G. Laurent, L. M. Hagelsieb, D. Lederer, P. E. Lober, D. Flandre, J. Remacle and J. P. Raskin: IEEE Solid-State Circuits Conference (2003) p. 337.

4. J. Li, C. Xu, Z. Zhang, Y. Wang, H. Peng, Z. Lu and M. Chan: Sens. Actuators, B 106 (2005) 378.

5. F. F. Bier, F. Kleinjung and F. W. Scheller: Sens. Actuators, B 38 (1997) 78.

6. R. de la Rica, F. S. Cesar and A. Baldi: Electrochem. Commun. 8 (2006) 1239.

7. S. J. Park, T. A. Taton and C. A. Mirkin: Science 295 (2002) 1503.

8. C. A. Mirkin: Inorg. Chem. 39 (2000) 2258.

9. C. Berggren, P. Stålhandske, J. Brundell and G. Johansson: Electroanalysis 11 (1999) 156.

10. L. A. Chrisey, G. U. Lee and C. E. O'Ferrall: Nucleic Acids Res. 24 (1996) 3031.

11. T. Andrew Taton, C. A. Mirkin and R. L. Letsinger: Science 289 (2000) 1757.

12. M. Ikeda, K. Nakazato, H. Mizuta, M. Green, D. Hasko and H. Ahmed: Nanotechnology $\mathbf{1 4}$ (2003) 123 . 